\title{
A Study of Interventions in Acute Pancreatitis at a Tertiary Care Hospital - An Indian Experience.
}

\author{
Deshpande AA ${ }^{1}$, Thanky $\mathrm{H}^{2}$, Soman $\mathrm{S}^{3}$. \\ ${ }^{1,2,3}$ Department Of Surgery, Seth G.S.Medical College \& K.E.M. Hospital, Maharashtra University Of Health \\ Sciences, India.
}

\begin{abstract}
:
Background: Acute pancreatitis is a common gastro-intestinal disease with varied etiology, presentations, management and outcomes. Over the years the management has evolved from surgical to conservative under intensive care setting. Here we have attempted to study various interventions in the management of acute pancreatitis and correlate clinical/radiological severity indices with likelihood of interventions and outcome.

Material And Methods: In this combined Retrospective-Prospective Observational study we have included 148 patients of diagnosed acute pancreatitis. Detailed history, clinical parameters, laboratory investigations, imaging findings, BISAP (Bedside Index for predicting Severe Acute Pancreatitis) score and Ctsi (Ct Severity Index), interventions, complications and outcome were recorded and analyzed.

Results: According to BISAP score, 72(49\%) had score of 0, 47(32\%) had 1, 22(15\%), 5(3\%) and 2(1\%) had scores of 2, 3 and 4 respectively while none had 5. CTSI was available for 112 patients out of which 94(84\%) had between 0-6 and 18(16\%) had 7-10. Initial conservative management was adequate in 119(80\%) patients. $29(20 \%)$ patients required some intervention: endoscopic (5\%), radiologic (8\%) or surgical (7\%). 9 patients required a second interventional treatment. Overall mortality was $10 \%$.

Statistically significant correlation was found between CTSI - likelihood of intervention/re-intervention and BISAP score - outcome (mortality) [ $p<0.001]$.

Conclusion: Aggressive conservative management remains the mainstay of treatment majority patients of acute pancreatitis. Tailored interventions are required in selected group of patients. CTSI correlates well with likelihood of intervention/re-intervention and BISAP score correlates well with outcome [mortality].
\end{abstract}

Keywords: Acute pancreatitis, Bedside Index for Severity of Acute Pancreatitis [BISAP score], CT Severity Index [CTSI], Interventions.

\section{Introduction}

Acute pancreatitis is a disease with varied etiology, presentation, complications, management strategies and outcomes depending on the severity and is a common gastro-intestinal disorder requiring acute hospital admission worldwide. Clinical course is usually mild, but $10 \%-20 \%$ patients suffer severe attack resulting in significant morbidity and mortality of up to $30 \%$.[1] Various severity scoring systems and prognostic indices have been described in the literature and a few used in clinical practice are Ranson's score,[2] APACHE II [Acute Physiology and Chronic Health Evaluation][3], BISAP [Bedside Index of Severity of Acute Pancreatitis][4] and Balthazar score/CTSI [CT Severity Index][5].

Few decades ago, early surgical intervention was attempted for severe pancreatitis with systemic complications. The utility of this strategy was questioned because of high mortality associated with the procedure. It was shown by a prospective randomized controlled trial comparing early (within 72 hours of symptoms) and late (at least after 12 days) necrosectomy that the mortality rates were $56 \%$ and $27 \%$ respectively leading to its premature termination.[6] Over the years, conservative management has become the mainstay in treatment of acute pancreatitis. The focus is on early fluid resuscitation and intensive monitoring. However, well planned and timely interventions are required in carefully selected group of patients. The timing of intervention has also changed from early necrosectomy to delayed interventions in cases with documented or suspected infection of pancreatic necrosis associated with clinical deterioration. The IAP/APA evidence based consensus guidelines are the most recent reference guidelines for management of acute pancreatitis ${ }^{7}$.[7]

We have attempted to study the interventions needed in the management of acute pancreatitis at our institute and also co-relate severity indices with likelihood of interventions and outcome. Our primary objective was to study the type, timing and frequency of interventions done in the management of acute pancreatitis.

\section{Material And Methods}

This is a combined Retrospective - Prospective Observational cohort study of patients of acute pancreatitis admitted in the Department of General Surgery at our institute over a period of 2 years. 148 patients of age more than 12 years with confirmed diagnosis of acute pancreatitis were included in the study. 
Clearance was obtained from the Institutional Ethics Committee. Patients' data regarding detailed history, examination findings, vital parameters, laboratory investigations, imaging findings were noted from the hospital papers. Details of their course in the ward, complications, treatment modalities used as well as details of any intervention required were also noted. We have included interventions directed at the primary pancreatic pathology only. Elective interval laparoscopic cholecystectomy was done for all the patients with gall stone induced pancreatitis, but has not been included as interventions studied. Ancillary procedures such as pleural tapping were also not included as interventions. The recorded BISAP scores for all the patients were noted and in the patients in whom CT scan was performed, CTSI was noted [N = 112].

As a post-hoc analysis we have studied correlation between BISAP score and CTSI with likelihood of interventions and outcome / mortality.

Demographic parameters were analyzed using appropriate descriptive statistics. Correlation between the severity indices and the need for interventions and outcome was analyzed using Chi square test.

\section{Results}

The age of patients presenting with acute pancreatitis in our study ranged from $13-62$ years. Almost $86 \%$ of our patients were between 21-50 years of age, out of which maximum [38\%] were from 31-40 age group. Female patients comprised $9 \%$ of the total study population. The most common etiology was found to be alcohol [68\%]. The etiological distribution of cases is depicted in fig. 1.

Distribution of the patients according to BISAP score and CTSI are given in Table 1 and 2 respectively.

Conservative management was adequate for in 119 patients [80\%]. 29 patients [20\%] required some intervention during their course of illness. Of these, $7 \%$ were surgical, $8 \%$ radiological and $5 \%$ endoscopic modalities. The details of primary interventions used are provided in Table 3 . Nine patients $[6 \%$ of total and $31 \%$ of patients with previous intervention] required second intervention during their admission course. Out of these 6 were surgical, 2 were radiological and 1 was endoscopic in nature as depicted in Table 4 .

Various loco-regional complications were: acute fluid collection in 33 patients, Pseudocyst in 18 patients, Portal vein/Splenic vein/Superior mesenteric vein thrombosis in 3 patients, and splenic artery pseudoaneurysm in 2 patients. Organ failure rate was 26.7\% [40 patients]; comprising acute renal failure [11], respiratory complications [25], central nervous system [3] and cardiovascular system [1].

Overall Mortality Rate was $6.75 \%$ [10 out of 148]. Seven from conservatively managed 119 patients [5.9\%] and 3 from 29 patients that required interventions [10.3\%] expired. 9 out of 10 patients expired from single or multi organ failure.

Among the 94 patients with CTSI $0-6,13$ [13.8\%] required intervention, while 8 out of 18 patients [44.4\%] with CTSI 7 - 10 required some intervention. This difference was found to be statistically significant by chi square test $[p<0.0001]$. Similarly 2 patients [2\%] from CTSI $0-6$ group required re-intervention as against 6 [33\%] in CTSI $7-10$ group which was again found to be statistically significant [p<0.0001]. All the 10 patients who had expired were from CTSI 0-6 group.

28 out of 141 patients [19.8\%] in BISAP score $0-2$ group required intervention as against 3 out of 7 [43\%] patients with BISAP $3-5$. This was not found to be statically significant by chi square test [p=0.113]. Similarly correlation between need for re-interventions and BISAP score was not statistically significant $[\mathrm{p}=0.318]$. BISAP score was found to be correlating well with mortality as it was observed that 5 out of 141 patients [3.54\%] with BISAP score of $0-2$ had expired against 5 out of 7 patients [71.4\%] with BISAP score 3 $-5[<0.001]$.

\section{Discussion}

Most of our patients are young men from productive age group and alcohol is the commonest causative factor. This is in contrast to the western population where the median age of a first attack of acute pancreatitis is in the fifth or sixth decade of life and proportion of women patients is larger.[8,9] According to the BISAP score 141 patients [95\%] were mild pancreatitis with score $0-2$ and 7 patients [5\%] were found to have severe pancreatitis that is score $3-5$. This distribution is more or less similar to other reported studies.[10,11] CTSI was available for 112 patients in which 94 patients [84\%] had mild pancreatitis with score $0-6$ against 18 patients [16\%] with score of $7-10$ were designated as having severe pancreatitis. The discrepancy of only $5 \%$ patients with BISAP score 3 and above but $18 \%$ of patients with CTSI 7 or above suggests that many of these patients would have qualified as moderately severe pancreatitis as per the newly proposed Revised Atlanta classification system.[12]

Despite the same initial conservative management, 29 patients [20\%] required interventions. The first intervention was radiological or endoscopic in $2 / 3^{\text {rd }}$ of the patients. Endoscopic Retrograde CholangioPancreaticography (ERCP) with Common bile duct (CBD) stenting was done for 7 cases of gall stone induced pancreatitis. All of them settled and did not require any further intervention during their admission. 
Ultrasound or CT guided pig tailing of collections / liquefied necrosis / infected pseudo cysts were done in 11 patients at an average of 10 days from the starting of symptoms. The idea behind image guided drainage is mainly to tide over the crisis by letting out the infected material and to buy time to let the necrosis get walled off or to improve the general condition of the patient. Five patients settled with the image guided drainage procedure alone and did not require any further interventions. However six patients required necrosectomy as second intervention later on during their course of illness in view of infection, clinical deterioration or persistent unwellness. These surgical interventions were done on an average 23 days after the onset of symptoms. Thus radiological interventions early in the course of multimodality management helped to delay surgical interventions till the fourth week when they can be performed more safely.

In the primary surgical interventions, one patient underwent distal pancreatectomy for traumatic pancreatitis. Among the remaining 9 patients, 5 patients were offered necrosectomy as first intervention at an average of 15 days from starting of symptoms. These were the patients in whom surgery was performed in view of clinical deterioration when image guided interventions could not be done due to unavailability of window, diffuse necrosis without localization or lack of resources. Malangoni et al[13] in their study of 60 patients of severe acute pancreatitis reported that all 13 patients of infected pancreatic necrosis and 8 patients of sterile necrosis having clinical deterioration, sepsis or worsening organ failure were managed with operative debridement, while 21 patients with sterile necrosis along with the rest 18 patients without necrosis were managed conservatively.

Another distinct group of 4 patients underwent cysto-gastrostomy or cysto-jejunostomy as first intervention at an average of 47 days from the starting of symptoms. These patients could withstand the initial acute inflammation phase without any interventions and were offered surgical internal drainage procedures when suitable wall thickness was attained.

Radiological procedures have also an important role as second interventions. Post necrosectomy, one patient underwent pigtailing of intra-abdominal collection and another one patient underwent Splenic artery embolization for pseudo-aneurysm. Thus each patient warrants an individually tailored management strategy utilizing various radiological, endoscopic and surgical interventions when required over and above the initial standard conservative management.

From the post-hoc analysis of our data, we have found a positive correlation between likelihood of interventions / re-interventions and the morphological severity of the disease demonstrated by CTSI. At the same time, CTSI is not a good predictor of the outcome of the disease in terms of mortality. This has been previously also emphasized in the literature that Balthazar scores are not significantly different between survivors and those who died ${ }^{13}$. On the contrary, disease outcome was more related to the patho-physiological severity of the disease as characterized by the BISAP score. Similar correlation has been demonstrated by VK Singh et $\mathrm{al}^{10}$ in their prospective evaluation of 397 cases of acute pancreatitis and concluded that the BISAP score represents a simple way to identify patients at risk of increased mortality. Papachristou et al ${ }^{11}$ have compared BISAP, Ranson's, APACHE-II, and CTSI scores in predicting organ failure, complications, and mortality in acute pancreatitis in 185 patients and concluded that the BISAP score is an accurate means for risk stratification in patients with acute pancreatitis and the prognostic accuracy of BISAP is similar to those of the other scoring systems.

There appears to be two distinct patterns of the disease process: the one with more morphological damage as depicted by higher CTSI score is more likely to undergo interventions but that may not necessarily result in poor outcomes, while the other one with more physiological damage as demonstrated by higher BISAP score is associated with poor prognosis. However this postulation requires further validation by large scale prospective studies.

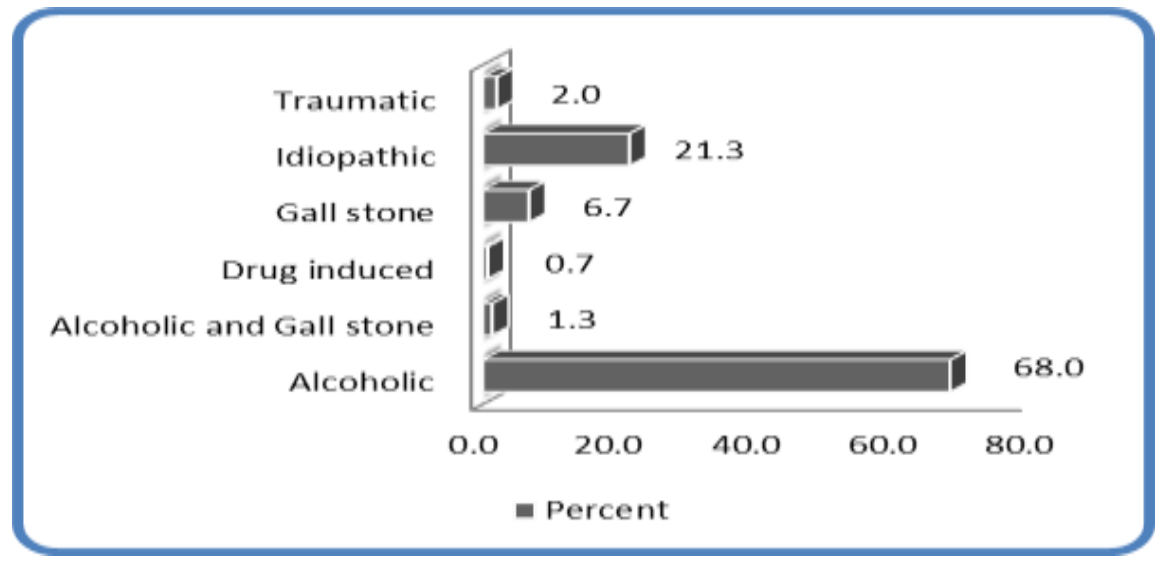

Figure 1. Etiological distribution of the study population. 
A study of interventions in acute pancreatitis at a tertiary care hospital - an Indian experience.

\begin{tabular}{|l|l|}
\hline BISAP score & No. of patients [\%] \\
\hline 0 & $72[48.6 \%]$ \\
\hline 1 & $47[31.8 \%]$ \\
\hline 2 & $22[14.9 \%]$ \\
\hline 3 & $05[3.4 \%]$ \\
\hline 4 & $02[1.3 \%]$ \\
\hline 5 & 0 \\
\hline & 148 Total \\
\hline
\end{tabular}

Table 1. Distribution of the study population as per BISAP score.

\begin{tabular}{|l|l|}
\hline CTSI & No. of patients[\%] \\
\hline 0 to 6 & $94[84 \%]$ \\
\hline 7 to 10 & $18[16 \%]$ \\
\hline & 112 Total \\
\hline
\end{tabular}

Table 2. Distribution of the study population as per CTSI.

\begin{tabular}{|l|l|}
\hline Intervention & No. of patients \\
\hline Necrosectomy & 05 \\
\hline Distal Pancreatectomy & 01 \\
\hline $\begin{array}{l}\text { Cysto-gastrostomy / } \\
\text { Cysto-jejunostomy }\end{array}$ & 04 \\
\hline Pigtailing of Necrosis / Pseudocyst / Collection & 11 \\
\hline Splenic artery Embolization & 01 \\
\hline ERCP with CBD stenting & 07 \\
\hline & $29[$ Total ] \\
\hline
\end{tabular}

Table 3. Primary interventions used in the treatment of acute pancreatitis.

\begin{tabular}{|l|l|l|}
\hline Re Intervention & No. of patients & Prior Intervention \\
\hline Necrosectomy & 6 & Pigtailing of collection / necrosis \\
\hline Pigtailing & 1 & Necrosectomy \\
\hline Splenic artery embolization & 1 & Necrosectomy \\
\hline ERCP with PD stenting & 1 & Pigtailing of pseudocyst \\
\hline & 9 [Total] & \\
\hline
\end{tabular}

Table 4. Re-Interventions used in the treatment of acute pancreatitis.

\section{Conclusion}

Acute pancreatitis is a disease of the young men with alcohol being the most common etiology in our community. Majority of hospital admissions are for mild and moderately severe attacks. Interventions are required in around $20 \%$ of patients. Well planned and tailored-to-need combinations of endoscopic, radiologic and surgical interventions play an important role in treating these patients. Morphological severity score - CTSI correlates well with likelihood interventions / re-interventions while patho-physiological severity score - BISAP score is better predictor of outcome in terms of mortality. BISAP is a simple and effective prognostic scoring system in Indian population also.

\section{Acknowledgements}

We wish to thank Dr. A.N. Supe, Director (ME \& MH) and Dean (Seth G. S. Medical College \& K.E.M. Hospital) for permitting us to publish hospital data.

\section{References}

[1]. Yadav D, Lowenfels AB. The epidemiology of pancreatitis and pancreatic cancer. Gastroenterology 2013; $144: 1252-61$.

[2]. Ranson JH, Rifkind KM, Roses DF, Fink SD, Eng K, Spencer FC . "Prognostic signs and the role of operative management in acute pancreatitis". Surgery, Gynecology \& Obstetrics 1974;139 (1): 69-81. PMID 4834279.

[3]. Knaus WA, Draper EA, Wagner DP, Zimmerman JE. APACHE II: a severity of disease classification system. Crit Care Med 1985; 13:818-29.

[4]. Wu B.U., Johannes RS, Sun X et al. The early prediction of mortality in acute pancreatitis: a large population-based study. Gut 2008; 57: 1698-703

[5]. Balthazar EJ, Robinson DL, Megibow AJ, Ranson JH . "Acute pancreatitis: value of CT in establishing prognosis". Radiology 1990; 174 (2): 331-336. PMID 2296641.

[6]. Mier J, Leon E, Castillo A, et al. Early versus late necrosectomy in severe necrotzing pancreatitis. Am J Surg 1997; 173:71-5.

[7]. Working Group IAP/APA Acute Pancreatitis Guidelines. Pancreatology 2013; 13: e1 - e15.

[8]. Goldacre MJ \& Roberts SE. Hospital admission for acute pancreatitis in an English population, 1963-98: database study of incidence and mortality. BMJ 2004; 328: 1466-1469. 
[9]. Frey CF, Zhou H, Harvey DJ et al. The incidence and case-fatality rates of acute biliary, alcoholic, and idiopathic pancreatitis in California, 1994-2001. Pancreas 2006; 33: 336-344.

[10]. Singh VK, Wu B.U., Bollen TL, Repas K, Maurer R, Johannes RS, Mortele KJ, Conwell DL, Banks PA. “A prospective evaluation of the bedside index for severity in acute pancreatitis score in assessing mortality and intermediate markers of severity in acute pancreatitis". Am J Gastroenterol. 2009 Apr; 104(4):966-71.

[11]. Papachristou GI, Muddana V, Yadav D, et al. Comparison of BISAP, Ranson's, APACHE-2 and CTSI scores in predicting organ failure, complications and mortality in acute pancreatitis. Am J gastroenterol. 2010; 105(2): 435-441.

[12]. Peter A Banks, Thomas L Bollen, Christos Dervenis, Hein G Gooszen, Colin D Johnson, Michael G Sarr et al. Acute Pancreatitis Classification Working Group. "Classification of acute pancreatitis-2012: revision of the Atlanta classification and definitions by international consensus". Gut 2013; 62:102-111.

[13]. M.A. Malangoni and A.S. Martin. Outcome of severe acute pancreatitis. The American Journal of Surgery 189 (2005) 273-277. 